\title{
THE THIRD-ORDER NONLINEAR OPTICAL SUSCEPTIBILITY IN OCTUPOLAR MOLECULE AND ITS DIPOLAR SUBUNIT
}

\author{
B. Derkowska ${ }^{a} *$, B. Sahraoui ${ }^{a} \dagger$, X. Nguyen Phu ${ }^{a}$ and C. Andraud ${ }^{b}$ \\ ${ }^{a}$ Laboratory POMA, EP CNRS 130 University of Angers \\ 2 Bd. Lavoisier, 49045, Angers Cedex 01, France \\ ${ }^{b}$ Ecole Normale Supérieure de Lyon, Stéréochimie et Interaction Moléculaires \\ UMR CNRS-ENS Lyon, 46, Allée d'Italie, 69364 Lyon Cedex 07, France
}

\begin{abstract}
The third-order nonlinear optical susceptibility $\chi^{\langle 3\rangle}$ of octupolar molecule with three double bonds was measured using degenerate four wave mixing technique at $532 \mathrm{~nm}$ in tetrahydrofuran solution. For comparison, we also measured its analogous dipolar subunit. We found that the $\chi^{\langle 3\rangle}$ value for octupolar molecule is about twice larger than its dipolar subunit.

PACS numbers: 42.65.An, 78.66.Qn
\end{abstract}

\section{Introduction}

The experimental and theoretical investigations have revealed that the organic materials show a large third-order optical nonlinearity, low optical losses, and a fast response. From that point of view they are promising materials for applications in photonics based technologies of optical signal processing, broad-band optical communications, integrated optics, optical sensing, optical computing [1-3], optoelectronics, and nonlinear optical devices [4].

Third-order nonlinear optical properties of dipolar molecules are widely investigated area of research. Recently, new octupolar molecules have been recognized as potentially useful for nonlinear applications. From that point of view, some experiments have been carried out to develop a better understanding of the relationship between molecular structure and nonlinear optical properties [5, 6].

In present paper, we report experimental investigations of the third-order nonlinear optical susceptibility $\chi^{\langle 3\rangle}$ of octupolar molecule and its corresponding dipolar subunit using the standard degenerate four wave mixing (DFWM) technique at $532 \mathrm{~nm}$.

\footnotetext{
${ }^{*}$ Also in Institute of Physics, Nicholas Copernicus University, Grudziądzka 5, 87-100 Torun, Poland.

$\dagger^{\dagger}$ corresponding author; e-mail: bouchta.sahraoui@univ-angers.fr
} 


\section{Experimental}

The DFWM response of these molecules was measured with a mode-locked $\mathrm{Nd}$ :YAG laser at $532 \mathrm{~nm}$. The pulse duration and the repetition rate were $30 \mathrm{ps}$ and $1 \mathrm{~Hz}$, respectively. The spatial profile of the laser pulse was Gaussian.

In DFWM measurement (Fig. 1), two counterpropagating strong beams $(\langle 1\rangle$ and $\langle 2\rangle)$ are incident on the sample with a third weaker probe beam $(\langle 3\rangle)$. The probe beam is incident at an angle $\theta\left(\theta=12^{\circ}\right)$ with respect to the pump beams. Two of these beams interfere inside the sample to form a grating from which the third beam diffracts to form a conjugate signal $(\langle 4\rangle)$ that retraces the probe path. The incident wave intensities verify the relations $I_{1}(z=0)=I_{2}(z=L)$ and $I_{3}=$ $6 \times 10^{-2} I_{1}$. The phase conjugated signal was detected by a photomultiplier tube (PM). A portion of the input beam was picked off and measured by a photodiode $\left(V_{c}\right)$ to monitor the input energy. The photodiode and conjugate signals were averaged and displayed by a Tektronix TDS 3054 Digital Phosphor Oscilloscope.

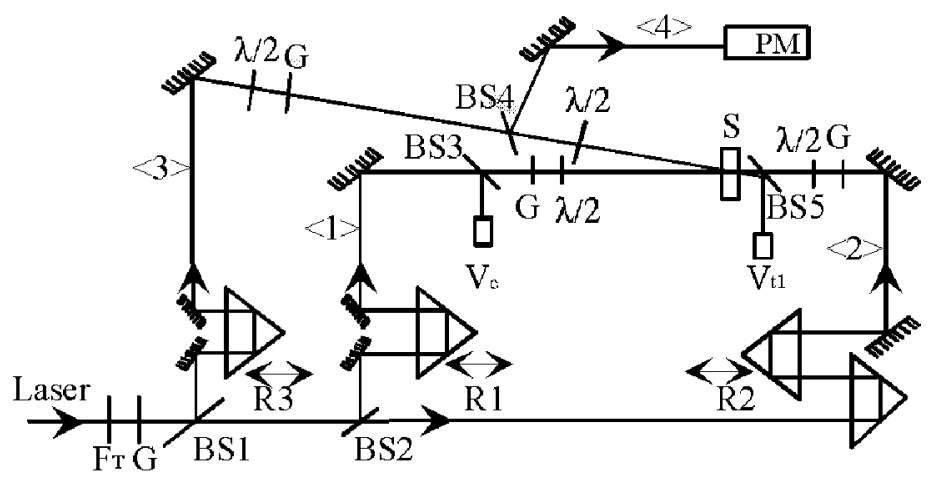

Fig. 1. Experimental setup: $\langle 1\rangle$ and $\langle 2\rangle-$ pump beam, $\langle 3\rangle-$ probe beam, $\langle 4\rangle-$ fourth beam, $\mathrm{S}$ - sample, $\mathrm{F}_{\mathrm{T}}$ - neutral filter, $\mathrm{R}_{1}, \mathrm{R}_{2}, \mathrm{R}_{3}$ - delay lines, $\mathrm{G}$ - glan prism, $V_{c}, V_{t 1}$ - control photodiodes, $\mathrm{PM}$ - photomultiplier tube, BS - beam splitter.

In the experiment, our compounds are studied in solution. We used tetrahydrofuran (THF) as a solvent for its high solubility with our molecules, and small third-order optical nonlinearity, which is neglected [7]. The measured third-order nonlinear optical susceptibility $\chi^{\langle 3\rangle}$ is the response, which comes from the sample solute. $\mathrm{CS}_{2}$ was used as a reference material for the measurement. The third-order nonlinear susceptibility of $\mathrm{CS}_{2}$ is estimated to be $\chi^{\langle 3\rangle}=2.1 \times 10^{-12} \mathrm{esu}$. Using our DFWM setup, only the magnitude (not the phase of the $\chi^{\langle 3\rangle}$ ) is obtained. In $\mathrm{CS}_{2}$ case, the $\chi^{\langle 3\rangle}$ is purely real, because there is no thermal grating and linear and nonlinear absorption at the laser frequency.

\section{Results and discussion}

The chemical structures of octupolar and dipolar molecules are shown in Fig. 2. The absorption spectra of studied compounds are presented in Fig. 3. It 

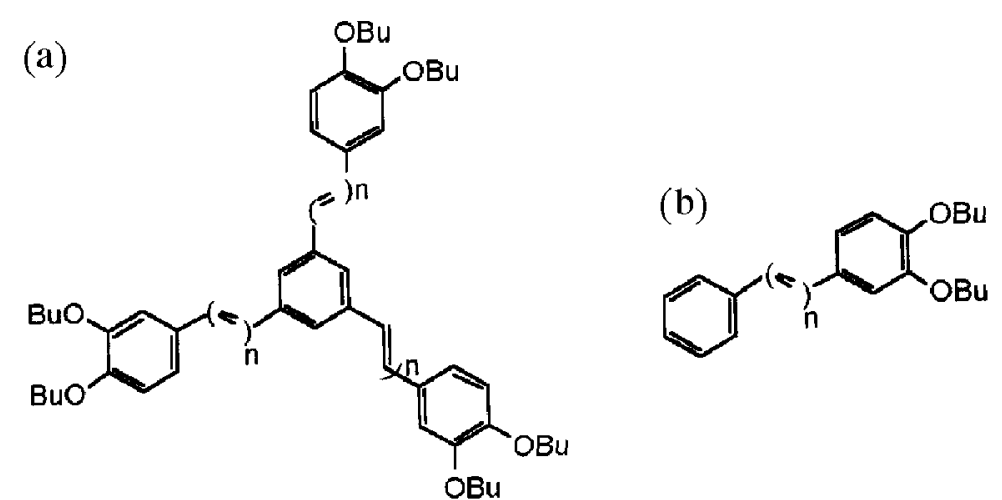

Fig. 2. The chemical structure of (a) octupole molecule and (b) its corresponding dipolar subunit.

is apparent from a comparison of these two spectra that the octupole absorption wavelengths are almost identical to those of its dipole analogues, only showing a small red shift. There is however a considerable difference in the oscillator strengths of those two classes of molecules, which are markedly enhanced in octupole with respect to the corresponding dipole [8].

These absorption spectra were made with $1 \mathrm{~cm}$ long cell and for the solutions at very weak concentration $\left(1 \times 10^{-5} \mathrm{M}\right)$. We can notice that both octupolar and dipolar molecules do not absorb at $532 \mathrm{~nm}$ of our DFWM measurements. It means that the predominant part of $\left|\chi^{\langle 3\rangle}\right|$ for octupole and its corresponding dipolar subunit at $532 \mathrm{~nm}$ is nonresonant.

The transmission measurements showed that the studied molecules present only linear absorption at $532 \mathrm{~nm}$. This indicates that the third-order nonlinear optical susceptibility of these materials is a real number at the excitation wavelength, so $\operatorname{Im} \chi^{\langle 3\rangle}=0$.

The experimental and theoretical results of DFWM reflectivity $R$ are presented in Fig. 4. The $\chi^{\langle 3\rangle}$ values for studied molecules were measured as a function of incident intensity. The DFWM reflectivity $R$ was calculated from the propagation equation of the interacting beams which are deduced from the Maxwell equations using the slowly varying amplitude approximation with taking into account linear absorption $\alpha$. The DFWM reflectivity $R$ can be expressed as follows [9]:

$$
R=\frac{I_{4}(0)}{I_{3}(0)}=\left(\frac{48 \pi^{3}}{n^{2} c \lambda} \chi^{\langle 3\rangle}\right)^{2} \frac{I_{1}(0) I_{2}(0) \exp (-\alpha L)}{\left[p \operatorname{coth}(p L)+\frac{\alpha}{2}\right]^{2}}
$$

where $p^{2}=\frac{\alpha}{4}-\left(\frac{48 \pi^{3}}{n^{2} e \lambda} \chi^{\langle 3\rangle}\right)^{2} I_{1}(0) I_{2}(0)$.

As can be seen in Fig. 4, the solid line shows the best fit with theoretical formula (1) to the experimental data. The real values of $\chi^{\langle 3\rangle}$ estimated from the dependencies of Fig. 4 and theoretical formula (1) are presented in Table. We can see that the $\chi^{\langle 3\rangle}$ value for octupole is larger than for its dipolar subunit. This may be due to the fact that the octupolar molecule is three times larger than its corresponding subunit. Second, there is probably some interaction, among the side 

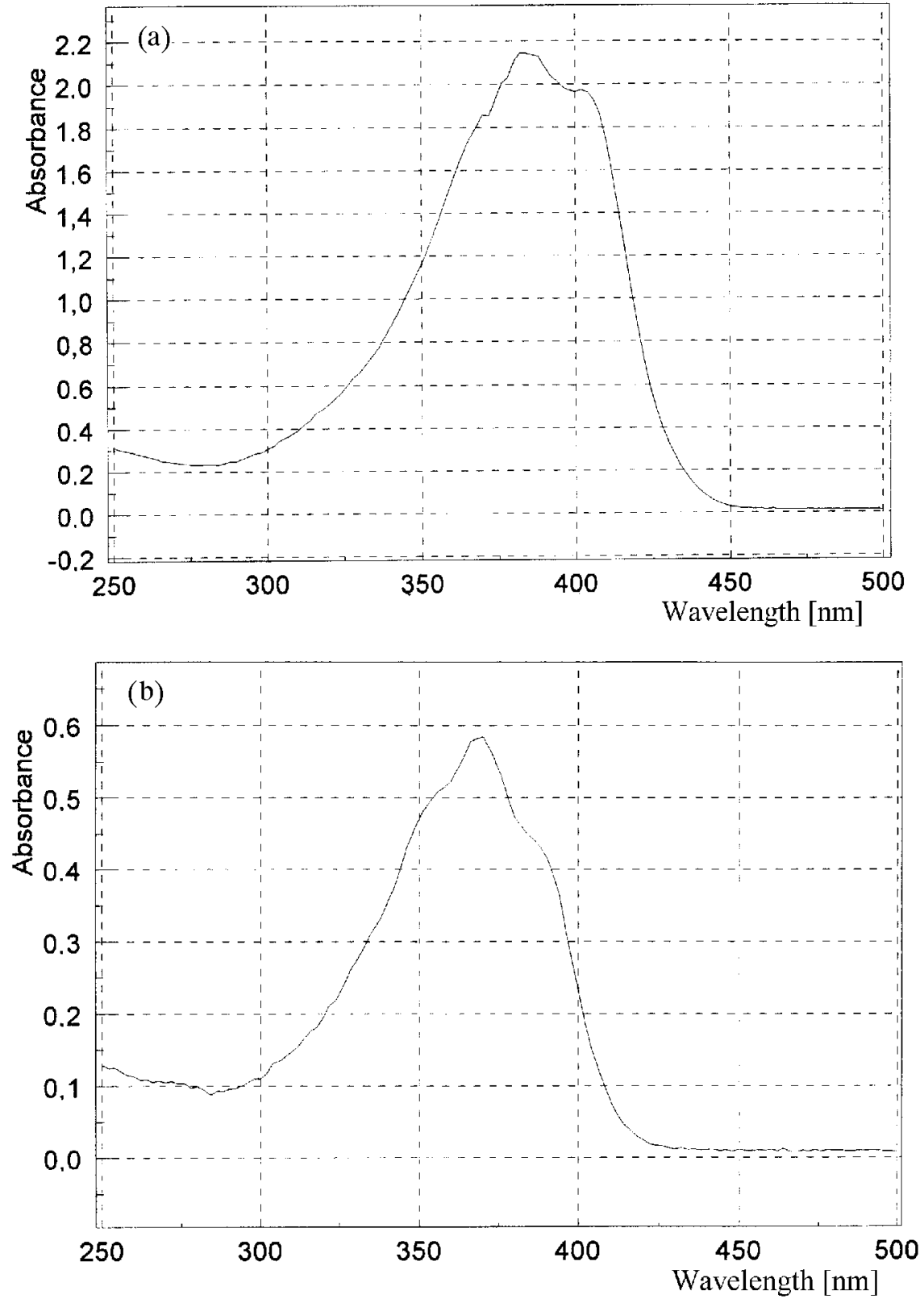

Fig. 3. The absorption spectra of (a) octupole molecule and (b) its corresponding dipolar subunit.

wings through the central molecule. We think that the charge transfer not only does occur from the peripheral donors towards the central ring, but also to some extent between the different branches of the molecule [8]. 


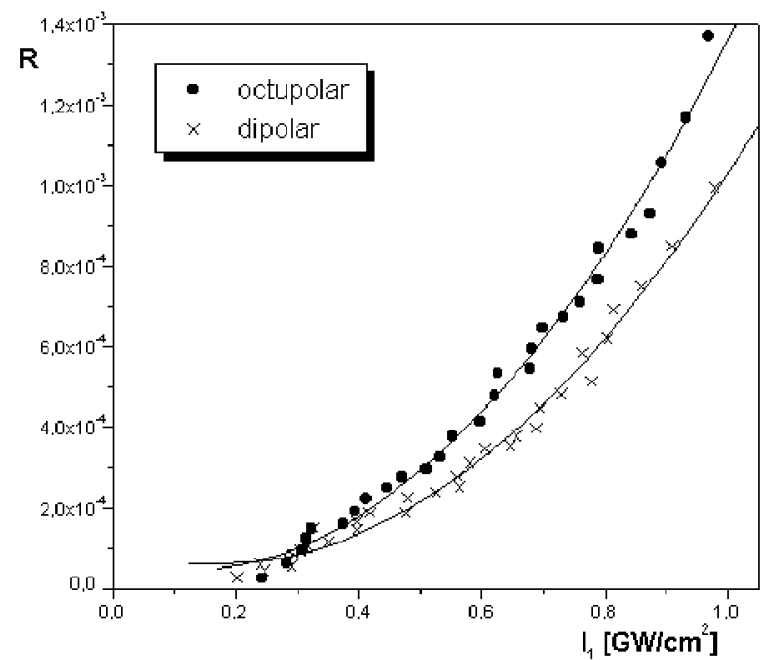

Fig. 4. The DFWM reflectivity $R$ as a function of incident light $I_{1}$ for octupolar $(\bullet)$ and dipolar $(x)$ molecules.

TABLE

The values of linear absorption $\alpha$ coefficients, the absolute value of third-order nonlinear optical susceptibility $\chi^{\langle 3\rangle}$ for octupolar molecule and its corresponding dipolar subunit.

\begin{tabular}{c|c|c|c}
\hline \hline Molecule & $\begin{array}{c}\alpha \\
{\left[\mathrm{cm}^{-1}\right]}\end{array}$ & $\begin{array}{c}\left|\chi^{\langle 3\rangle}\right| \times 10^{21} \\
{\left[\mathrm{~V}^{2} \mathrm{~m}^{-2}\right]}\end{array}$ & $\begin{array}{c}\chi^{\langle 3\rangle} \times 10^{19} / \alpha \\
{\left[\mathrm{V}^{2} \mathrm{~m}^{-1}\right]}\end{array}$ \\
\hline dipole & 3.24 & 6.79 & 2.09 \\
octupole & 3.36 & 8.84 & 2.63
\end{tabular}

The third-order nonlinear optical properties of a given material can be also characterized as a figure of merit, $\chi^{\langle 3\rangle} / \alpha$, which is related to the inherent strength of the nonlinear process. Based on the data, which are presented in Table, the figure of merit of octupole is about twice larger than that of its dipolar subunit.

\section{Conclusions}

In this paper, we investigated the third-order nonlinear optical susceptibility $\chi^{\langle 3\rangle}$ of octupolar molecule and its corresponding dipolar subunit in THF using DFWM technique at $532 \mathrm{~nm}$.

The DFWM reflectivity $R$ for octupolar and dipolar molecules was measured as a function of incident laser powers. The third-order nonlinear optical susceptibility $\chi^{\langle 3\rangle}$ for studied molecules was calculated. We found that the $\chi^{\langle 3\rangle}$ value of octupolar molecule appears to be twice larger than its dipolar subunit. The reason for this could be the fact that there is interaction among the side wings through 
the central molecule, which leads to a longer absorption wavelength of octupole compared to its dipolar subunit. On the other hand, the size of octupole is about three times larger than that of dipole.

We can observe that the octupole possesses relatively large third-order optical nonlinearities and the small linear absorption $\alpha$ which means that it possesses a good figure of merit $\left(\chi^{\langle 3\rangle} / \alpha\right)$. Materials with such good figure of merit could be promising materials for the molecular engineering of new nonlinear optics molecules.

\section{References}

[1] Characterization Technique and Tabulations for Organic Nonlinear Optics Materials, Eds. M.G. Kuzyk, G.W. Dirk, Marcel Dekker, Inc., New York 1998.

[2] Organic Thin Films for Waveguiding Nonlinear Optics, Eds. F. Kajzar, J.D. Swalen, Gordon and Breach Pub., Amsterdam 1996.

[3] Kwang-Sup Lee, Oh-kil Kim, Photonics Science News 4, 9 (1999).

[4] S. Wang, W. Huang, T. Zhang, H. Yang, Q. Gong, Y. Okuma, M. Horikiri, Y.F. Miura, Appl. Phys. Lett. 75, 1845 (1999).

[5] P.N. Prasad, D.J. Williams, Introduction to Nonlinear Optical Effects in Molecules and Polymers, Wiley, New York 1991.

[6] Nonlinear Optical Properties of Organic Materials and Crystals, Eds. J. Zyss, D. Chemla, Academic Press, New York 1987.

[7] B. Sahraoui, X. Nguyen Phu, M. Sallé, A. Gorgues, Opt. Lett. 23, 1811 (1998).

[8] C. Andraud, T. Zabulon, A. Collet, J. Zyss, Chem. Phys. 245, 243 (1999).

[9] B. Sahraoui, R. Chavalier, G. Rivoire, J. Zaremba, M. Sallé, Opt. Commun. 135, 109 (1997) 